\title{
Same Patient, Same Site: Using Different Topical Medicine for Psoriasis
}

\author{
Yi-Guo Feng* and Xiao-Min Gao
}

Department of Dermatology, The Second Affiliated Hospital, Xi'an Jiaotong University, China

Dear editor,

Topical use is the main advantage for dermatological therapy due to the outer-layer features of skin. Consequently, numerous preparations are available for the dermatosis. However, the efficacy evaluation of different preparations accurately is always very difficult. Psoriasis is a common skin condition characterized by persistent erythematous scaly plaque. It is estimated about $3 \%$ population suffered from this notorious calcitrant disease. The traditional and accepted method (until now it is also popular in practice). To observe treatment effect of cream is to apply one kind of drug to a group of patients with a specific disease who received different systemic approaches that might influence the effect of topical appliance [1]. Here we show 3 very severely affected psoriatic patients, with several years of history, whose lumber regions were marked into six different areas (Figure 1, Figure 2 , and Figure 3) being applied with six different kinds of cream (Figure 4), respectively in the following seven days. The topical cream was spread, and clinical pictures were taken by a designated nurse each day. The patients did not know which

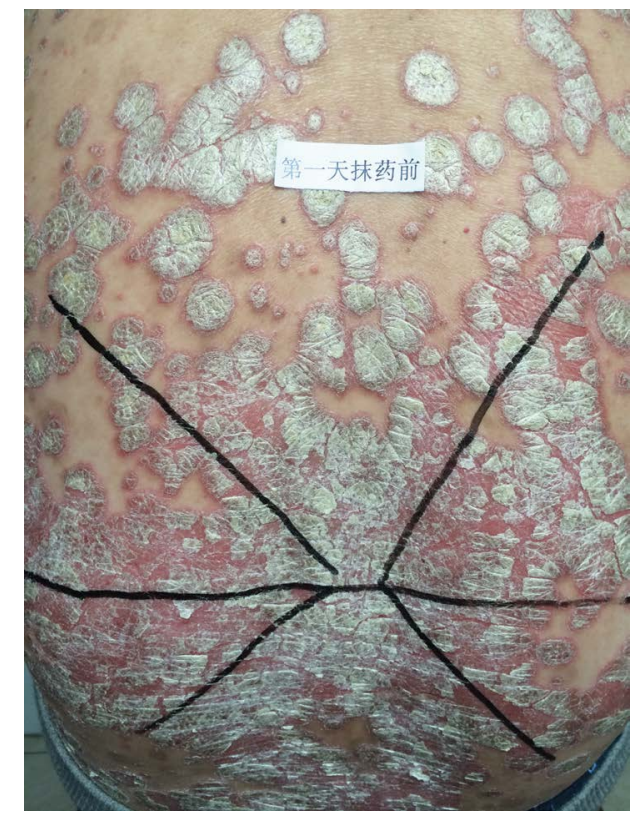

Figure 1: The first day before topical application. The Chinese characters in the picture have the same meaning as such. cream be used to which area, so they are blinded. The creams include $0.1 \%$ tacrolimus ointment (Protopic, Astellas), $0.1 \%$ tazarotene cream (China Chongqing Huapont Pharm. Co. LTD), calcipotriol ointment (Bright Future), 0.1\% mometasone furoate cream (Eloson, Bayer) and two hospital self-made creams (one is piyanjin cream containing clobetasol, the other vehicle cream named danchun cream without any medicine component). On the third day before topical appliance (Figure 5), the fourth day (Figure 6) and the seventh day (Figure 7), different efficacy could be seen clearly. To our great surprise, most of the creams have similar effects, even the vehicle

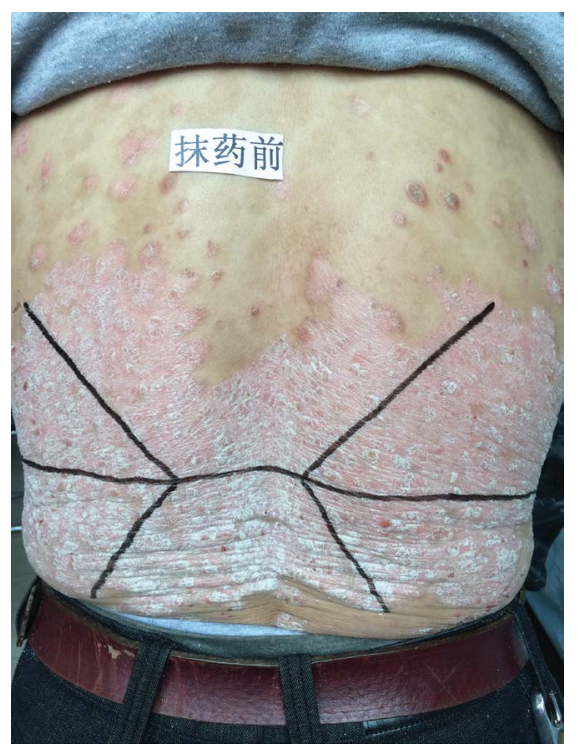

Figure 2: The first day before topical application. The Chinese characters in the picture have the same meaning as such.

*Corresponding author: Yi-Guo Feng, Department of Dermatology, The Second Affiliated Hospital, Xi'an Jiaotong University, 157 Xiwu Road, Xi'an, China

Accepted: November 20, 2018

Published online: November 22, 2018

Citation: Yi-Guo F, Xiao-Min G (2018) Same Patient, Same Site: Using Different Topical Medicine for Psoriasis. Dermatol Arch 2(1):72-73

Copyright: (c) 2018 Yi-Guo F, et al. This is an open-access article distributed under the terms of the Creative Commons Attribution License, which permits unrestricted use, distribution, and reproduction in any medium, provided the original author and source are credited. 


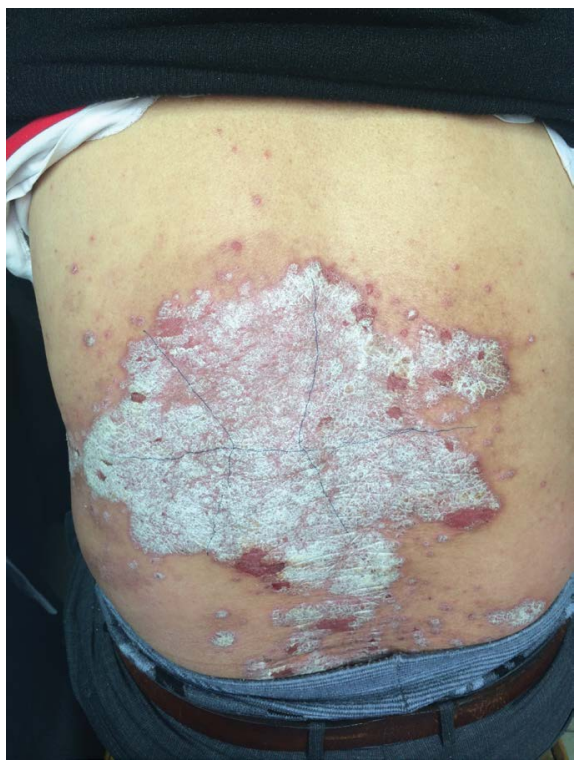

Figure 3: The first day before topical application.

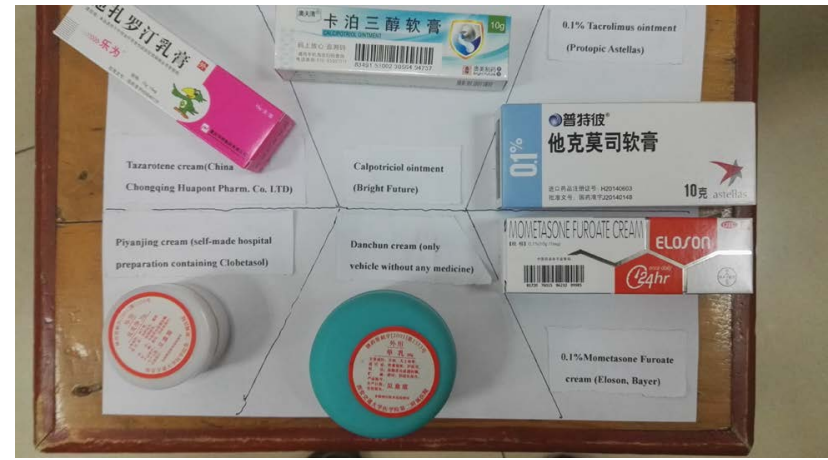

Figure 4: Six different topical medicine corresponding to six areas on the patients.

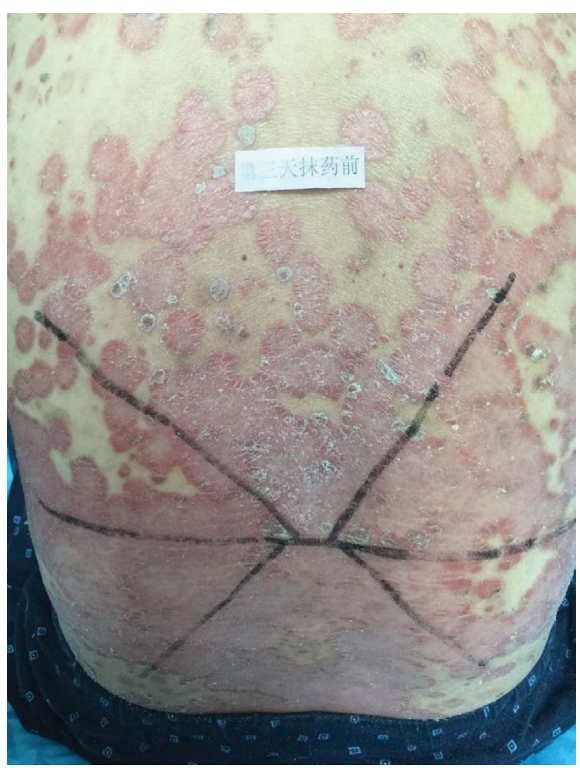

Figure 5: The second day after one application. Note the marked improvement.

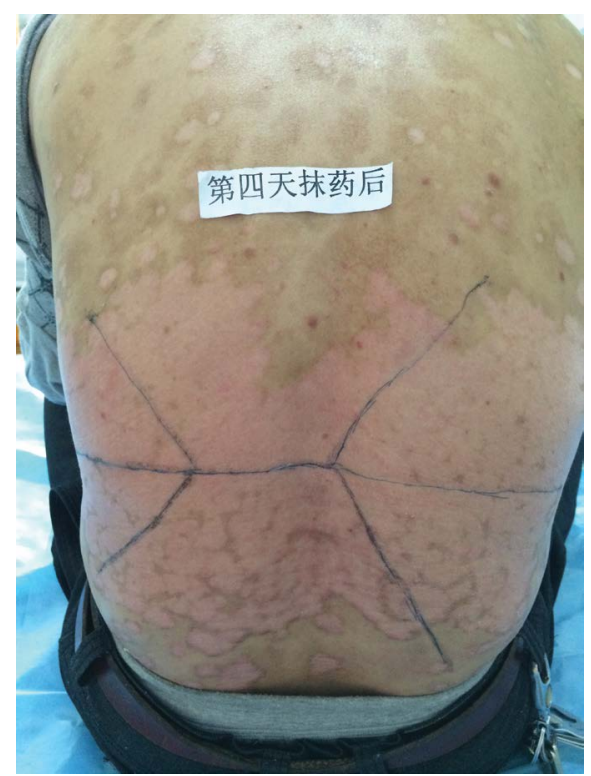

Figure 6: The fourth day after four applications. Note the remarkable clearance of scaling.

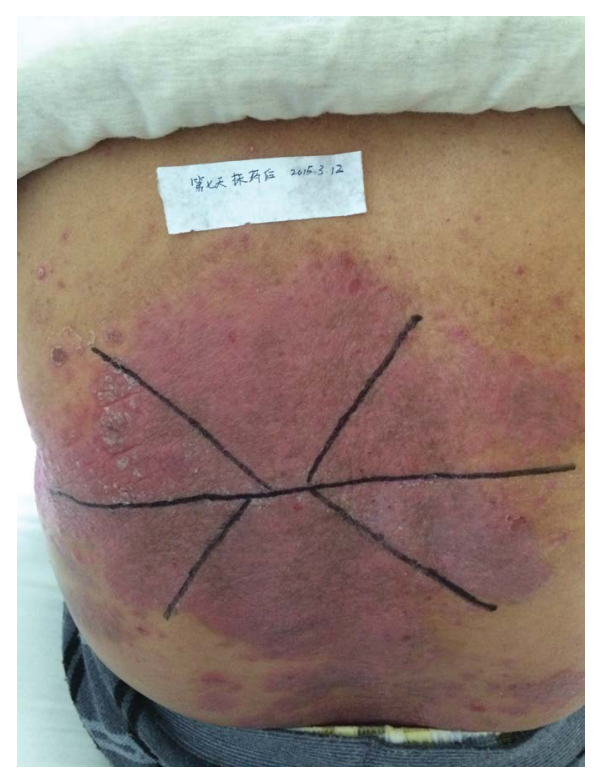

Figure 7: The $7^{\text {th }}$ day after seven applications. Note the nearly completely clearance of the lesion.

cream without any medicine component. This method avoids the influences of different systemic medicine, patients' personal response, anatomical sites and disease duration on the efficacy evaluation of topical preparations. We coined the SPSS rule which stands for the acronym of same patient, same site or symmetrical site for different creams. We propose it be implemented in the future evaluation of topical preparations.

\section{References}

1. Kerdel FA, Draelos ZD, Tyring SK, et al. (2018) A phase 2, multicenter, double-blind, randomized, vehicle-controlled clinical study to compare the safety and efficacy of a halobetasol propionate $0.01 \%$ lotion and halobetasol propionate $0.05 \%$ cream in the treatment of plaque psoriasis. J Dermatolog Treat 5: 1-7. 